\title{
Kardiopulmonale Reanimation von Schwangeren
}

Prof. Dr. med. Alexander Strauss, Frauenarzt, Kiel

Ein Herzkreislaufstillstand kommt bei Schwangeren zwar selten vor, er kann jedoch für Mutter und Kind tödlich enden. Entscheidend für das Überleben von Mutter und Kind sind die Qualität und die Geschwindigkeit, in der Wiederbelebungsmaßnahmen durchgeführt werden. Aspekte wie Fachwissen, Teamarbeit, Simulationstraining spielen eine wichtige Rolle für den Erfolg der Reanimation. Hebammen sind in besonderem Maße gefordert.

\section{Herzkreislaufstillstand bei Schwangeren}

Die Inzidenz kardialer Akutereignisse ist zwar abhängig von Lebensalter und Geschlecht, trotzdem sind auch Frauen im reproduktionsfähigen Alter betroffen.

Einen Kreislaufkollaps erleiden schwangere Frauen mit einer Häufigkeit von 0,3-0,6\%. Meist ist eine Verminderung des venösen Rückstroms zum Herzen, bedingt durch das reversible Vena-CavaKompressionssyndrom verantwortlich. Dieser vital nicht bedrohliche Zustand ist durch

- konsequente Linksseitenlagerung,

- $\mathrm{O}_{2}$-Gabe $(100 \%)$,

- Blutdruck-Monitoring (Hypotension $<100 \mathrm{mmHg} \rightarrow$ verminderte Plazenta-Perfusion),

- bedarfsgerechte Infusion zur Vorlasterhöhung (i.v.-Zugang oberhalb des Zwerchfells) und

- die Identifikation und Beseitigung der Ursachen

ohne Schaden für Mutter und Kind zu beherrschen [1].

Ein Herzkreislaufstillstand (Sudden Cardiac Arrest, SCA) ist an den Leitsymptomen Asystolie - Blutdruckabfall - Bewusstlosigkeit - blasses Hautkolorit erkennbar.
Die Prävalenz ist durch die Zunahme anamnestischer mütterlicher Risikokonstellationen und durch die Zunahme von Schwangerschaftskomplikationen in den vergangenen Jahren gestiegen. Aktuell liegt die Prävalenz bei 1/12.000 Schwangeren [1].

Tab. 1 zeigt die Ursachen für einen Herzkreislaufstillstand bei Schwangeren.

Zur raschen Ursachenklärung kann der 4H-4T-Algorithmus eingesetzt werden (Tab. 2). Dieser Algorithmus zeigt die reversiblen Ursachen für einen Herzkreislaufstillstand. Jedes dieser Ereignisse kann zum plötzlichen Herztod führen, der häufigsten indirekten mütterlichen Todesursache, und bedarf des unmittelbaren, koordinierten und multidisziplinären Einsatzes des Notfallteams [2].

Entscheidend für das mütterliche und das fetale Outcome ist die Geschwindigkeit und die Qualität, mit der schwangerschaftsadaptierte Wiederbelebungsmaßnahmen implementiert, kontrolliert eskaliert und interdisziplinär koordiniert werden. Trotz des potenziell fatalen Ereignisses ist eine mütterliche Gesamtüberlebensrate von bis zu 59\% zu erreichen, auch weil Schwangere peripartal von Hebammen engmaschig kontrolliert werden [1].

\section{Kardiopulmonale Reani- mation der Schwangeren}

Fachwissen, Teamarbeit, Wachsamkeit

Die Lebensgefährdung von Mutter und Kind ist bei kritischen Herzkreislauferkrankungen immanent. Diejenigen Medizinprofessionen, die diese seltenen Notfälle bewerten und beherrschen müssen, benötigen hierfür spezialisiertes Fachwissen gepaart mit großer Wachsamkeit. Außerdem ist eine multidisziplinäre und multiprofessionelle Teamarbeit notwendig.

Kardio-respiratorische Funktionen und (Labor-)Parameter passen sich physiologisch den Bedürfnissen der Schwangerschaft an. Diese Anpassung beeinflussen die Diagnostik und Therapie eines Herzkreislaufstillstands bei Schwangeren im Vergleich zur Reanimation bei NichtSchwangeren [3]. Da durch die Schwangerschaft auch die klinische Einschätzung verändert wird, ist die Kenntnis dieser Veränderungen (Tab. 3) besonders wichtig, um Fehleinschätzungen und damit den Verlust wertvoller Zeit zu vermeiden [4].

Mit dem Ziel, möglichst rasch die adäquaten mütterlichen Herzkreislaufpara- 
meter wiederherzustellen, entsprechen die Empfehlungen zur kardiopulmonalen Reanimation von Schwangeren den Richtlinien der Erwachsenenreanimation, abgesehen von wenigen Aspekten.

\section{Richtlinien}

Die allgemeingültigen Richtlinien zu Wiederbelebungsmaßnahmen (European Resuscitation Council, ERC-Guideline) weisen unter anderen Sondersituationen auch Anpassungen an die Situation Schwangerschaft auf $[1,5,6,7]$.

Tab. 1 Ätiologische Faktoren eines Herzkreislaufstillstands bei Schwangeren.

\begin{tabular}{|c|c|}
\hline \multicolumn{2}{|l|}{ Ursachen } \\
\hline $\begin{array}{l}\text { Kardiovaskuläre } \\
\text { Ursachen }\end{array}$ & $\begin{array}{l}\text { - Koronare Herzkrankheit (ca. } 80 \%) \\
\text { - Nicht-ischämische Kardiomyopathie (10-15\%) } \\
\text { - Strukturelle/funktionelle Herzerkrankungen (<5\%) }\end{array}$ \\
\hline $\begin{array}{l}\text { Vorübergehende } \\
\text { Auslöser }\end{array}$ & $\begin{array}{l}\text { - Elektrolytstörung } \\
\text { - Hypoxie } \\
\text { - Azidose } \\
\text { - Stress, erhöhter Sympathikotonus } \\
\text { - Vagus-Reizung } \\
\text { - Antiarrhythmika } \\
\text { - Pharmakologische Ursachen (Oxytocin, Magnesium, } \\
\text { Opiode, Insulin, Anaphylaxie, Medikamentenverwechslung, } \\
\text { Drogen) }\end{array}$ \\
\hline $\begin{array}{l}\text { Arrhythmie- } \\
\text { Mechanismen }\end{array}$ & $\begin{array}{l}\text { - Reentry-Phänomene (kreisende Erregungen) } \\
\text { - Autonome Schrittmacherzentren }\end{array}$ \\
\hline $\begin{array}{l}\text { Extrakardiale } \\
\text { Faktoren }\end{array}$ & $\begin{array}{l}\text { - Anästhesiologische Komplikationen (hohe neuroaxiale } \\
\text { Blockade, Hypotension, Beatmungsproblematik, Aspiration, } \\
\text { Hypoventilation, systemische Toxizität von Lokalanästhetika) } \\
\text { - Thrombose } \\
\text { - Embolie } \\
\text { - Blutung (z. B. Koagulopathie, uterine Atonie, vorzeitige } \\
\text { Plazentalösung, Plazenta praevia, Uterusruptur, Trans- } \\
\text { fusionsreaktion) } \\
\text { - Infektion, Fieber, Sepsis } \\
\text { - Trauma, Selbstmord } \\
\text { - Spannungspneumothorax } \\
\text { - Psychiatrische Störung } \\
\text { - Schock }\end{array}$ \\
\hline $\begin{array}{l}\text { Schwangerschafts- } \\
\text { spezifische Ursachen }\end{array}$ & $\begin{array}{l}\text { - Schwangerschaftsbedingte hypertensive Erkrankung } \\
\text { (Präeklampsie, Eklampsie, HELLP-Syndrom) } \\
\text { - Fruchtwasserembolie } \\
\text { - Peripartale Kardiomyopathie } \\
\text { - Ektope Schwangerschaft }\end{array}$ \\
\hline
\end{tabular}

\section{Rasche Erfassung und effektive} Ausschaltung reversibler Ursachen

- Unmittelbare Diagnostik der Ursachen für den Herzkreislaufstillstand (Tab. 1). Da die häufigsten Ursachen schwangerschaftsbedingt sind, ist die geburtshilfliche Expertise essentiell.

- Gezielte, schwangerschaftsadaptierte therapeutische Intervention

- Bei einem Herzkreislaufstillstand, der im schwangeren Zustand als irreversibel eingeschätzt wird und einer Schwangerschaftsdauer $>24$ SSW $\rightarrow$ Perimortem Kaiserschnitt ohne weiteren Zeitverzug

\section{Optimierung des Effekts der \\ Herzdruckmassage}

- Die optimale Position zur Durchführung der bimanuellen Herzdruckmassage entspricht der bei nicht-schwangeren Personen (Zentrum des Brustkorbs, unabhängig von schwangerschaftsbedingter Verdrängung/ Zwerchfellhochstand)

- Die Thoraxkompression mit einer Tiefe von 5-6 cm und einer Frequenz von (100-)120 Kompressionen pro Min. bei fehlender bzw. minimaler Unterbrechung (Lagerung, Diagnostik, Perischock-Pause < 10 Sek.) erreicht eine vergleichbare Effektivität wie bei nicht-schwangeren Personen [6, 7].

\section{Beachtung des Vena-Cava- Kompressionssyndroms}

Das Vorkommen des Vena-Cava-Kompressionssyndroms nimmt mit höherem Gestationsalter zu. Es kann das Herzzeitvolumen um bis zu 2/3 reduzieren.

- Bei Linksseitenlagerung der Patientin um $27^{\circ}$ (Keilkissen) können - verglichen zur Rückenlage - für die kardiopulmonale Reanimation nur $80 \%$ der Thoraxkompressionskraft erreicht werden [8]. Daher ist die Reanimation in Rückenlage (feste Unterlage) vorzunehmen. Außerdem wird der Winkel der Kippung der Patientin meist überschätzt: $<20^{\circ} \rightarrow$ keine Verbesserung des venösen Rückflusses, $>30^{\circ} \rightarrow$ Gefahr des Abrutschens/Rollens der Patientin. Der Einsatz von Keilkissen vermeidet diese Fehleinschätzung.

- Die ununterbrochene manuelle Linksverlagerung des Uterus (optimal in Zweihand-Technik) in Rückenlage der Patientin verbessert den venösen Rückstrom zum Herzen aus der unteren Körperhälfte und übertrifft die Wirkung einer Linksseitenlagerung der Patientin. Außerdem optimiert dieses Vorgehen die Effektivität der kardiopulmonalen Reanimation während der Herzdruckmassage inkl. Beatmung in Rückenlage bzw. während einer perimortalen Schnittentbindung (bis zur Kindsentwick- 
Tab. 2 4H-4T-Algorithmus der reversiblen Ursachensuche eines Herzkreislaufstillstandes.

\begin{tabular}{|l|l|}
\hline $\mathbf{4 ~ H}$ & $\mathbf{4 ~ T}$ \\
\hline Hypovolämie & Tabletten \\
\hline Hypoxie & Tamponade (kardial) \\
\hline Hyper-/Hypokaliämie, H-lonen (Azidose) & Tension (Spannungspneumothorax) \\
\hline Hypothermie & Thromboembolie pulmonal, koronar \\
\hline
\end{tabular}

lung) - Beginn ohne Zeitverlust und Druckeinschränkung [9].

- Perimortem Kaiserschnitt: unmittelbare Sectiobereitschaft herstellen und ggf. konsequent zeitnahe Durchführung (kein Transport der Patientin, um Zeitverlust und Reduktion der Effektivität der Reanimation zu vermeiden).

\section{Kompliziertes Atemwegsmanagement}

Die Progesteron-vermittelte laryngeale/ tracheale Ödemneigung in der Schwangerschaft muss berücksichtigt werden.

- Anpassung der Tubusgröße bei der Intubation (Innendurchmesser der Trachea um 0,5-1 mm reduziert)

- Höhere Komplikationsrate/Gefahr der Fehlintubation durch Hyperämie, Hypersekretion, Schleimhautödem und schwierigere Arbeitsbedingungen durch die (Links-)Seitenlage (Anatomie der Atemwege stellt sich anders dar als in Rückenlage)

- Gesteigertes Aspirationsrisiko (gastrointestinaler Reflux, Kardiainsuffizienz)

- Entsättigung während Apnoe-Phasen erfolgt rascher als bei Nicht-Schwangeren.

\section{Der fetale Zustand muss in der} Akutsituation diagnostisch und therapeutisch außer Acht bleiben

- Als unzulässig zeitverbrauchend (bis auf Einzelfälle) ist eine geburtshilfliche (Ultraschall-) Diagnostik von Aspekten wie Geburtsfortschritt, Blutung, Schwangerschaftskomplikationen und die fetale Zustandsbeurteilung anzusehen und daher zu unterlassen.
- Die kardiopulmonale Reanimation bzw. die perimortale Sectio darf nicht verzögert/behindert werden.

\section{Defibrillation bei Schwangeren}

- Die gleiche Impedanz bei schwangeren wie bei nicht-schwangeren Frauen (biphasische Defibrillationsenergie: 120-200 Joule, eskalierend)

- Die fetale Exposition der elektrischen Energie ist minimal = keine Gefahr für das Kind (Stromfluss durch den Uterus/Fruchtwasser, maßgeblich sind Stromstärke und Kontaktdauer)

- Die veränderte Anatomie in der Schwangerschaft erschwert den Paddeleinsatz $\rightarrow$ Klebeelektroden verwenden

- Eine Lichtbogenbildung zu externen oder internen fetalen Überwachungseinheiten ist unwahrscheinlich (Einzelfallberichte), deshalb müssen sie während des Stromeinsatzes nicht entfernt werden. Somit wird eine Verzögerung der Defibrillation vermieden.

\section{Pharmakologische Intervention}

- Keine schwangerschaftsspezifischen Anpassungen bei der Indikation und Dosierung trotz erhöhter glomerulärer Filtrationsrate (renale Durchblutung $\uparrow 40 \%$ ) und gesteigertem Plasmavolumen ( $\uparrow 40-50 \%)$ in der Schwangerschaft (Tab.3).

\section{Arbeit im erweiterten Team}

- Teamzusammensetzung:

- Notfallmediziner/Anästhesist/ Internist/Chirurg und Intensivpflegepersonal/Anästhesiepflegepersonal/Pflegepersonal
- Geburtshilflicher Anästhesist und Anästhesiepflegepersonal

- Geburtshilflicher Teambestandteil: Hebamme und Geburtshelfer/ggf. Pflegepersonal

- Neonatologischer Teambestandteil: Neonatologe und neonatologisches (Intensiv)Pflegepersonal

- Situationsabhängig alternierende Teamführung durch Notfallmediziner, Geburtshelfer und Neonatologen

Implementierung von institutionsspezifischen Checklisten für seltene kritische Notfallsituationen

Die Empfehlungen zur Reanimation von Schwangeren sind nur zum Teil ausreichend wissenschaftlich fundiert. Die praktischen Regeln zur Durchführung einer Herzdruckmassage (manuelle Tätigkeiten, Lagerung in Rückenlage und manuelle (Links-)Lateralisierung des Uterus), die Kenntnisse zur Defibrillation und der Nutzen einer perimortem durchgeführten Sectio sind durch Studiendaten abgesichert $[6,7,8]$. Da Situationen, in den Schwangere reanimiert werden müssen, selten vorkommen, fehlen zu allen übrigen Empfehlungen der kardiopulmonalen Reanimation belastbare Studiendaten. Die publizierten Leitlinien basieren hier entweder auf Daten von nicht-schwangeren Frauen, auf Daten aus Simulationsstudien oder geben Expertenmeinungen wieder [8]. Dies betrifft z.B. die Empfehlung zur Anwendung (Indikation wie Dosis) von Adrenalin (1 mg alle 3-5 Min.) oder Amiodaron (300 mg) nach 3-malig erfolgloser ventrikulärer Defibrillation (tachykarde Rhythmusstörung, Kammerflimmern) und zur Fibrinolyse bei lebensbedrohlicher massiver Lungenembolie. Eine Lysebehandlung darf bei Schwangeren zwar nicht ohne Bedenken angewendet werden (Gefahr von Blutungen, fetaler Hypoxie), muss jedoch im indizierten Fall - trotz eines mütterlichen Mortalitätsrisikos von $1 \%$ - als potenziell lebensrettend zum Einsatz kommen [6, 7]. 
Tab. 3 Physiologische Anpassungsreaktionen des mütterlichen Organismus während der Schwangerschaft.

\begin{tabular}{|c|c|c|c|c|c|}
\hline \multicolumn{2}{|c|}{ Herz-/Kreislaufsystem } & \multicolumn{2}{|l|}{ Atmung } & \multicolumn{2}{|l|}{ Blutgerinnung } \\
\hline $\begin{array}{l}\text { Mittlerer arterieller } \\
\text { Blutdruck (Vasodila- } \\
\text { tation: Progesteron, } \\
\text { Östrogen, NO) }\end{array}$ & $\begin{array}{l}\downarrow 4-15 \% \\
\text { (Nadir im II. Trimenon) }\end{array}$ & Alveoläre Ventilation & $\uparrow 70 \%$ & \multicolumn{2}{|c|}{ Renale Ausscheidung } \\
\hline Plasmavolumen & $\uparrow 40-50 \%$ & Atem-Minuten-Volumen & $\uparrow 50 \%$ & & \\
\hline $\begin{array}{l}\text { Herzzeitvolumen } \\
(\mathrm{HZV})\end{array}$ & $\uparrow 30-50 \%$ & $\begin{array}{l}\text { Funktionelle Residualkapazität } \\
\text { (verdrängungsbedingt) }\end{array}$ & $\downarrow 10-25 \%$ & & \\
\hline \multirow{6}{*}{$\begin{array}{l}\text { Uteroplazentare } \\
\text { Perfusion } \\
(50 \rightarrow 1000 \mathrm{ml})\end{array}$} & & $\begin{array}{l}\mathrm{O}_{2} \text {-Verbrauch (im III. Trimenon } \\
\text { durch Bedarf von Mutter und } \\
\text { Fetus) }\end{array}$ & $\uparrow 20-33 \%$ & & \\
\hline & & $\mathrm{paO}_{2}$ & $\uparrow 10 \mathrm{mmHg}$ & & \\
\hline & & $\mathrm{paCO}_{2}$ & $\downarrow 10 \mathrm{mmHg}$ & & \\
\hline & & Hypokapnie & bis $32 \mathrm{mmHg}$ & & \\
\hline & & Milde respiratorische Alkalose & & & \\
\hline & & $\begin{array}{l}\text { Rechtsverschiebung der Sauer- } \\
\text { stoffbindungskurve }\end{array}$ & $\mathrm{P}_{50} 27 \rightarrow 30 \mathrm{mmHg}$ & & \\
\hline
\end{tabular}

Quelle: Strauss A., Sanders L., Ohnesorge H., Gräsner J.T. Geburtshilfliche Notfälle III - Präklinische Geburtshilfe. Der Notarzt 2013

\section{Qualitätsmanagement}

Die Bewältigung eines Herzkreislaufstillstands einer Schwangeren ist auch im Krankenhaus trotz des Einsatzes multiprofessioneller Interventionsteams mit einer Fehlerquote von $\mathbf{2 9} \%$ behaftet. Diese Irrtümer wirken sich auch auf die Überlebensraten aus [2]. Im Einzelnen tragen infrastrukturellen Faktoren zu 75\% der mütterlich fatalen Ausgänge bei. Kommunikationsdefizite im Team sind dagegen in $70 \%$ bezogen auf neonatale Morbidität und Mortalität als (mit)verantwortlich anzusehen [1].

Aufgrund der Seltenheit und der prognostischen Tragweite dieser Ereignisse fordert der European Resuscitation Council die Herstellung einer „institutionellen Bereitschaft“ der Klinik im Umgang mit
Herzkreislaufstillstand und kardiopulmonaler Reanimation bei Schwangeren. Er empfiehlt regelmäßige interdisziplinäre Teamtrainingsmaßnahmen $\mathrm{zu}$ Diagnose, Monitoring und koordinierendem Management von (geburtshilflichen) SCA-Patientinnen. Uniprofessionelles Training führte mitunter sogar zur Verschlechterung der Leistung des Gesamtteams [2].

Antrainierte Erfahrungen bei der Ersterkennung wie auch Fähigkeiten zur unmittelbaren Initiierung einer fachgerechten Reanimation sind, aufgrund ihres Verantwortungsbereiches in der Geburtshilfe in besonderem Maße von Hebammen gefordert.

\section{Spezifische Aufgabengebiete}

In der Reanimation von Schwangeren treten charakteristische Probleme auf:

- Zeitverlust bei der Wahrnehmung des Herzkreislaufstillstandes, bedingt durch die Schwangerschaft

- Zeitverzögerung bis zum Beginn der kardiopulmonalen Reanimation, bedingt durch die Schwangerschaft

- Erhöhte Raten ineffektiver Herzdruckmassagen (no-flow), bedingt durch die Schwangerschaft

- Häufige Vernachlässigung von Maßnahmen zur venösen Rückstromverbesserung

- Kommunikationsdefizite im Team aufgrund der ungewohnten Zusammensetzung 
- Unfähigkeit des Teams zur kontinuierlichen Fokussierung auf die kritisch erforderlichen Aufgaben

- Deutliche Unterschiede zwischen den Ergebnissen individueller Wissensüberprüfungen und den praktischen Fähigkeiten von Reanimationsteammitgliedern (nicht nur bei den geburtshilflich Beteiligten) [10]

Folgende Faktoren sind entscheidend für den Erfolg der Reanimation in außergewöhnlichen Situationen (wie die Reanimation einer Schwangeren): Inhaltsqualität und Struktur der Team(aus)bildung: Evidenzbasierung, Wirklichkeitsnähe, Multiprofessionaltität, kontinuierliches Monitoring

Die Qualität der Teamleistung zeigt sich an diesen Zielgrößen:

- Effektivität der kardiopulmonalen Reanimation

- Nachhaltigkeit der Teamarbeit, maßgeblich für den Erfolg ist hierbei das fachlich optimale Zusammenwirken aller Teilnehmer

- Stärkung von Team- und Führungskompetenzen (frühzeitige Diagnosestellung, koordinierende Aufgabenverteilung).

\section{Zwei Leben}

Perimortem Sectio caesarea bei mütterlichem Herzkreislaufstillstand

Der European Resuscitation Council (ERC) wie auch die American Heart Association (AHA) geben in ihren Empfehlungen neben den Vorgaben zum Basic life support (BLS) während der Schwangerschaft auch Instruktion zur Durchführung von Maßnahmen des Advanced life support (ACLS) vor $[1,6]$ :

Bei nicht erfolgreichen Reanimationsbemühungen über eine Dauer von 4 Min. soll die Indikationsstellung zur Perimortem Sectio erfolgen (gemäß European Resuscitation Council) [6, 7].
Die operative Entwicklung des Kindes per Schnittentbindung nach einer weiteren Minute soll dabei den venösen Rückstrom zum mütterlichen Herzen begünstigen und Dank der mechanischen Entlastung der Vena cava inferior die Erfolgsaussichten der Wiederherstellung normaler mütterlicher Kreislaufparameter verbessern $[6,7]$.

Bedingung ist ein Setting (Ort, Logistik), in dem eine unmittelbare Notfallentbindung möglich ist. Primäre Zielgröße ist eine rasche Erhöhung der mütterlichen Herzauswurfleistung (10\% bei Kompression der Vena cava inferior vs. $30 \%$ ohne Beeinträchtigung des zentralvenösen Rückflusses zum Herzen).

Durch die Eröffnung der Bauchhöhle besteht außerdem die Möglichkeit, die Aorta zu komprimieren und eine offene Herzdruckmassage durchzuführen.

Da ein längerfristiges intrauterines Überleben des Feten nur durch die Wiederherstellung einer mütterlichen Herzkreislaufstabilität gewährleistet werden kann, ist der, aus maternaler Sicht absolut indizierte Perimortem Kaiserschnitt unter Postponierung all seiner Effekte auf das kindliche Befinden/Prognose vorzunehmen. Als sekundäre mütterliche Interventionsziele der PMCD kommen durch die Eröffnung der Bauchhöhle mögliche werdende Kompressions-/ Klemmmöglichkeiten der Aorta und die Option der offenen Herzdruckmassage zum Tragen.

Die Diagnostik der fetalen Situation (Lebenszeichen, Versorgung) ist verzichtbar und darf, wenn in dieser Situation überhaupt vorgenommen, dabei die Reanimation der Mutter keinesfalls verzögern [2].

Nichtsdestotrotz nimmt das Schicksal des Kindes, und dies vorwiegend in $\mathrm{Ab}$ hängigkeit seines Gestationsalters, Einfluss auf die Eingriffseinschätzung (Indikationsimpakt)/-logistik (Lokalisationsimpakt) eines Perimortem Kaiserschnitts [1]:
- <20. SSW: Keine perimortale Sectio caesarea, da der Uterus zu klein ist, um den venösen Rückstrom klinisch wesentlich zu behindern. Keine Berücksichtigung der fetalen Prognose.

- 20.-24. SSW: Die Indikation zur perimortalen Sectio basiert ausschließlich auf dem Ziel der Verbesserung des mütterlichen Reanimationsergebnisses. Keine Berücksichtigung der fetalen Prognose.

- > 24. SSW: Bestimmend für die Indikation bleibt der mütterliche Herzkreislaufstillstand. Ein potenzielles Überleben des Kindes und damit die Erfordernis einer akuten neonatologischen Betreuung/Reanimation ist als Option zu berücksichtigen.

Bestimmend für die fetale Morbidität und Mortalität ist neben dem Schwangerschaftsalter die potenzielle Dauer der intrauterinen Hypoxie (mütterlicher Asystolie). Eine zeitnahe Entbindung eröffnet auch bei nicht-reanimierbarer Mutter die einzige Möglichkeit für das Kind zu überleben und trägt in diesen Fällen u. U. auch zur Vermeidung neurologischer Spätfolgen bei.

Der Reanimationserfolg bei der Mutter ist maßgeblich vom Zeitintervall zwischen Herzkreislaufstillstand und Perimortalem Kaiserschnitt abhängig. Die höchste Überlebenswahrscheinlichkeit der Mutter und ggf. auch des Kindes wird bei einer Entbindung innerhalb von $\mathbf{5}$ Minuten nach Herzkreislaufstillstand beschrieben. Tatsächlich findet die Entwicklung des Kindes in der Praxis allerdings meist deutlich später statt $[5$, $8,9]$.

\section{Behandlungspfade für den Notfall praktisch umsetzen}

Diese Aspekte müssen in der Praxis verwirklicht sein:

- System für die Erstentscheidung bzw. für die Triage

- Strukturen für die bedarfsgerechte Alarmierung 
Tab. 4 Externes multiprofessionelles/interdisziplinäres Simulationstrainingskonzept.

\section{Notärztliches/rettungsdienstliches - Notfallmanagement bei Schwangeren}

- Erstentscheidung - Triagierung

- Transportziel (nächstgelegene Geburtsklinik oder Perinatalzentrum?)

- Wohin innerklinisch mit der geburtshilflichen Notfallpatientin? (Notaufnahme - Schockraum - Kreißsaal - Operationssaal?)

- Aufbau spezifischer Notfallteams unter Würdigung der zentralen Rolle der Hebamme

- Festlegung und Koordination der internen und externen Patientinnentransportziele

- Nachhaltigkeit von internen Trainingsmaßnahmen

- Etablierung von institutionsspezifischen Handlungsalgorithmen (elektronisch, schriftlich, geschult)

- Regelmäßige Durchführung extern geleiteter multiprofessioneller| interdisziplinärer Simulationstrainings zur Koordinierung diagnostisch/therapeutischer Managementautomatismen, der Schaffung und des Ausbaus von internen und auswärtigen Kommunikationsstrukturen (geburtshilfliche Notfallhotline) (Tab.4).

\section{Literatur}

1 Jeejeebhoy FM, Zelop CM, Lipman S, Carvalho B, Joglar J, Mhyre JM, Katz VL, Lapinsky SE, Einav S, Warnes CA, Page RL, Griffin RE, Jain A, Dainty KN,
Herzkreislaufstillstand - Management während der Schwangerschaft

- Reanimation der Schwangeren

- Perimortem Sectio caesarea

- Teambildung

- Kommunikation

- Handlungsalgorithmen circumstances: Electrolyte abnormalities, poisoning, drowning, accidental hypothermia, hyperthermia, asthma, anaphylaxis, cardiac surgery, trauma, pregnancy, electrocution. Resuscitation. 2010; 81 (10): 1400-1433

7 King SE, Gabbott DA. Maternal cardiac arrest-rarely occurs, rarely researched. Resuscitation. 2011; 82 (7): 795-796

8 Einav S, Kaufman N, Sela HY. Maternal cardiac arrest and perimortem caesarean delivery: evidence or expertbased? Resuscitation. 2012; 83 (10): 1191-1200

9 Kundra P, Khanna S, Habeebullah S, Ravishankar M. Manual displacement of the uterus during caesarean section. Anaesthesia. 2007; 62 (5): 460-465

10 Hepp H. Zwei Leben - Anspruch und Wirklichkeit. Archives of Gynecology and Obstetrics. 1995; 257 (1): XVIIXXXVI

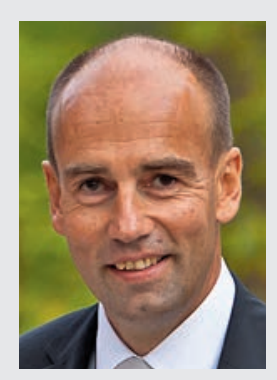

6 Soar J, Perkins GD, Abbas G, Alfonzo A, Barelli A, Bierens JJ, Brugger H, Deakin CD, Dunning J, Georgiou M, Handley AJ, Lockey DJ, Paal P, Sandroni C, Thies KC, Zideman DA, Nolan JP. European Resuscitation Council Guidelines for Resuscitation 2010 Section 8. Cardiac arrest in special
Univ.-Prof.

Dr. med. Alexander Strauss Christian-Albrechts-Universität zu Kiel

E-Mail: astrauss@email.uni-kiel.de 